\title{
Impact of an Infection Control Program on the Prevalence of Nosocomial Infections at a Tertiary Care Center in Switzerland
}

\author{
Corina Ebnöther, MD; Beate Tanner, MD; Flavia Schmid, MD;
}

Vittoria La Rocca, RN; Ivo Heinzer, MD; Thomas Bregenzer, MD

\begin{abstract}
oвjeCtive. To study the impact of a multimodal infection control program on the rate of nosocomial infections at a 550-bed tertiary care center.

METHODs. Before and after the implementation of an infection control program, the rate of nosocomial infection was recorded in timeinterval prevalence studies. Hand hygiene compliance was studied before and after the intervention. As a surrogate marker of compliance, the amount of alcohol-based hand rub consumed before the intervention was compared with the amount consumed after the intervention. The intervention included additional staff for infection control, repeated instructions for hand hygiene, new guidelines for preoperative antibiotic prophylaxis, and isolation of patients infected or colonized with multidrug-resistant bacteria.
\end{abstract}

RESULTS. The rate of nosocomial infection decreased from approximately $11.7 \%$ to $6.8 \%$ in 2 years. The rate of hand hygiene compliance increased by $20.0 \%$; it was $59.0 \%$ before the intervention and increased to $79.0 \%$ afterward. These results correlate with data on the consumption of alcohol-based hand rub, but not with data on the use of antibiotics.

CONCLUSION. Within 2 years, a multimodal infection control program intervention such as this one may reduce the rate of nosocomial infection at a tertiary care center by more than one-third and improve both the quality of care and patient outcomes. It may also generate considerable savings. Therefore, such programs should be promoted not only by hospital epidemiologists but also by hospital administrators.

Infect Control Hosp Epidemiol 2008; 29:38-43

For more than 150 years, nosocomial infection has been recognized as an important threat to hospitalized patients, ${ }^{1}$ one which causes up to 15,000 deaths annually in Great Britain ${ }^{2}$ and has enormous economic consequences. ${ }^{3,4}$ The impact of infection control in hospitals on patients' safety, the quality of care, and healthcare costs has been demonstrated in several publications. ${ }^{5,6}$ Thus, infection control is not only an economic issue but, even more importantly, it is an ethical obligation.? The cost effectiveness of infection control programs is well documented. ${ }^{2,3,6}$ Infection control in hospitals depends on clinicians' awareness of epidemiologic observations in wards and their awareness of findings in the microbiology laboratory. Cooperation and communication between microbiologists, hospital epidemiologists, and clinical infectious diseases specialists is crucial for effective infection control. An approximately one-third reduction in the incidence of nosocomial infections is an achievable goal if an infection control unit is staffed with 2-4 full-time infection control professionals per 500 beds, as recommended. ${ }^{5,8}$ Prior to the infection control interventions, the rate of nosocomial infection at our institution was $11.7 \%$, and the rate has been documented since 1999 in prevalence studies carried out in cooperation with the Swiss Nosocomial Infection Prevalence (SNIP) studies. ${ }^{9}$ In contrast to the above-mentioned staffing recommendation, at our 550-bed institution, only 1 full-time infection control nurse and a microbiologist $(20 \%$ of whose job time was dedicated to infection control) were responsible for infection control until 2004. On April 1, 2004, a full-time hospital epidemiologist was employed, and an infection control program was initiated. We analyzed the impact of this program on the prevalence of nosocomial infection from April 1, 2004, through June 30, 2006.

\section{METHODS}

We performed an observational study of the impact of an infection control program at Kantonsspital Aarau, a 550-bed tertiary care center in Aarau, Switzerland. On April 1, 2004, a full-time hospital epidemiologist was employed to implement this program. A new division for hospital hygiene and infection control that cooperated closely with the microbiologist and the infectious diseases clinician was established. Studies of the program's efficacy were conducted in 2006.

From the Division for Infection Control (C.E., V.L.), the Microbiology Laboratory (I.H.), and the Division of Infectious Diseases (B.T., F.S., T.B.), Kantonsspital Aarau, Switzerland.

Received July 24, 2007; accepted September 25, 2007; electronically published November 30, 2007.

(C) 2007 by The Society for Healthcare Epidemiology of America. All rights reserved. 0899-823X/2008/2901-0006\$15.00. DOI: 10.1086/524330 
TABLE 1. Consumption of Alcohol-Based Hand Rub During the 2-Year Study Period, by 6-Month Intervals

\begin{tabular}{lcccc}
\hline Variable & Jul-Dec 2004 & Jan-Jun 2005 & Jul-Dec 2005 & Jan-Jun 2006 \\
\hline Amount of hand rub consumed, L & $4,967.9$ & $4,611.2$ & $6,089.2$ & $6,494.9$ \\
Change from the previous period, L (\%) & $\ldots$ & $-356.7(-7.2)$ & $1,478(32.1)$ & $405.7(6.7)$ \\
\hline
\end{tabular}

NOTE. The hand hygiene intervention took place between September and December 2005.

The main steps of the infection control program were as follows.

\section{Managing Patients Colonized and/or Infected with Multidrug-Resistant Bacteria}

This intervention involved the revision of guidelines for the management and isolation of patients who were infected and/ or colonized with multidrug-resistant bacteria, such as methicillin-resistant Staphylococcus aureus (MRSA), extended-spectrum $\beta$-lactamase (ESBL)-producing gram-negative rods, aminoglycoside-resistant Pseudomonas aeruginosa, and vancomycin resistant enterococci (VRE). All isolates recovered from patients infected and/or colonized with MRSA were carefully analyzed and the strains were compared by pulsed-field gel electrophoresis (PFGE). The revised guidelines were communicated to all healthcare workers (HCWs), and instruction in infection control was provided for all HCWs. The infection control nurse was responsible for providing infection control instructions and ensuring that isolation guidelines were followed in wards that had patients in isolation.

\section{Preoperative Antibiotic Prophylaxis}

This intervention involved analysis of the guidelines for preoperative antibiotic prophylaxis for surgical patients and the implementation of a new guideline, which was distributed on pocket cards. Before this intervention (ie, in January 2003) and afterward (ie, in November 2004), we retrospectively analyzed 1,000 anaesthesia protocols from surgical procedures to determine the rate of compliance with the guideline that was in place at the time.

\section{Bacteremia Intervention}

Previous prevalence studies had demonstrated that up to $19.4 \%$ of nosocomial infections at our hospital were bloodstream infections (BSIs). Pittet et al. ${ }^{10}$ showed that the attributable mortality rate for nosocomial BSI is as high as $35 \%$ for intensive care unit patients, and the importance of early appropriate antibiotic treatment for these infections has been shown in studies by Leibovici et al. ${ }^{11}$ Therefore, in 2004, all patients with bacteremia were visited and assessed by the infectious diseases clinician. BSI was classified either as nosocomial or not nosocomial, in accordance with Centers for Disease Control and Prevention guidelines. In addition, a written recommendation for antibiotic therapy was given to the physician responsible for each case patients with bacteremia.

\section{Hand Hygiene Education and the Distribution of Hand Rub}

All HCWs and cleaning personnel were repeatedly instructed on hand hygiene. Alcohol-based hand rub was made easily available, and additional dispensers were installed on the walls in patients' rooms and at the door of each room. In addition, $100-\mathrm{mL}$ bottles were distributed for HCWs to carry in their pockets. This hand hygiene program was enhanced by participation in the Swiss Hand Hygiene Campaign (SHHC) in September 2005. ${ }^{12}$ As part of this campaign, hand-hygiene compliance was recorded according to the SHHC protocol both before and after the intervention. In addition, beginning in 2004, the hospital pharmacy recorded the consumption of alcohol-based hand rub at 6-month intervals.

\section{Timing of Studies and Analysis of Data}

In $1999,2002,2003$, and 2004, our institution participated in the SNIP studies, ${ }^{9}$ which were nationwide, 1-week prevalence studies. To control for the efficacy of our interventions, in 2006 we repeated the nosocomial infection prevalence study using the same method and case report forms as were used in the national studies. Data were analyzed at the SNIP study center in Geneva. Categorical data were compared using the $\chi^{2}$ test. A 2 -sided $P$ value of less than .05 was considered to be statistically significant.

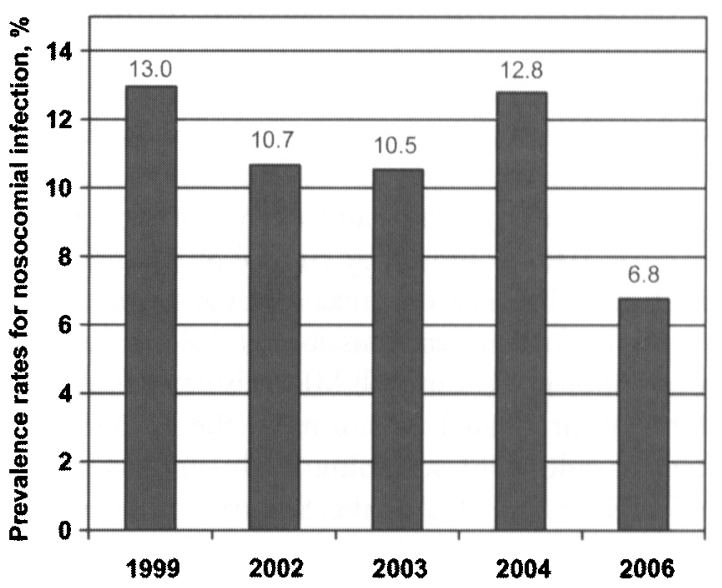

FIGURE. Prevalence of nosocomial infection from 1999 to 2006. In the years prior to 2004 the mean rate of nosocomial infections was $11.7 \%$. After implementation of the infection control program in 2004 , the rate of nosocomial infections dropped to $6.8 \%$ (OR, 0.51 [95\% confidence interval, $0.27-0.88$ ]; $P=.01$ ). 
TABLE 2. Estimates of the Impact of Nosocomial Infection Before and After Implementing an Infection Control Program in 2004

\begin{tabular}{|c|c|c|c|c|c|c|}
\hline \multirow[b]{2}{*}{ Variable } & \multicolumn{5}{|c|}{ Year } & \multirow{2}{*}{$\begin{array}{l}\text { Improvement, } \\
\text { estimated no. of } \\
\text { cases prevented } \\
\text { per year }\end{array}$} \\
\hline & 1999 & 2002 & 2003 & 2004 & 2006 & \\
\hline No. of patients & 270 & 253 & 332 & 328 & 310 & $\ldots$ \\
\hline No. $(\%)$ of patients with nosocomial infection & $35(13.0)$ & $27(10.7)$ & $35(10.5)$ & $42(12.8)$ & $21(6.8)$ & $\ldots$ \\
\hline Estimated no. of nosocomial infections per year & 1,820 & 1,404 & 1,820 & 2,184 & 1,092 & 715 infections \\
\hline $\begin{array}{l}\text { Estimated no. of deaths per year due to } \\
\text { nosocomial bacteremia }{ }^{b}\end{array}$ & 21 & 26 & 16 & 57 & 10 & $\cdots$ \\
\hline $\begin{array}{l}\text { Estimated no. of deaths per year due to all } \\
\text { nosocomial infections }{ }^{\text {b }}\end{array}$ & 91 & 70 & 91 & 110 & 55 & 35 deaths \\
\hline
\end{tabular}

\section{RES ULTS}

Before the intervention to improve hand hygiene, hand hygiene compliance was observed in accordance with the standardized protocol of the SHHC, as described by Sax and Pittet. ${ }^{12,13}$ The compliance rate (defined as a percentage of hand-hygiene opportunities completed appropriately) was $59.0 \%$ for the entire hospital, and compliance rates ranged from $42.1 \%$ to $78.3 \%$ among different wards. Hand hygiene compliance increased to $79.0 \%$ after the intervention and repeated instruction in hand hygiene (range, 65.4\%-100.0\% compliance). The percentage of improvement in hand hygiene compliance in various wards ranged from $2.4 \%$ to $42.7 \%$. Simultaneous with the improvement in hand hygiene compliance, the consumption of alcohol-based hand rub throughout the hospital increased by $32.2 \%$ after the intervention (Table 1 ).

The rate of infection and/or colonization with MRSA at our institution has been slowly increasing, but it is still below $2 \%$. PFGE was performed for all strains recovered from patients at our institution. In 2005, there was an outbreak of MRSA colonization that involved 1 patient and $2 \mathrm{HCWs}$ in a single ward. All patients and HCWs in the ward were screened for MRSA carriage by culture of nasal and pharyngeal swab samples. The outbreak was controlled after a few days, and no further transmission of MRSA has yet been recorded at our institution. All MRSA isolates recovered after this outbreak originated from outside the hospital and were detected by culture of screening swab samples obtained at admission. Less than $1 \%$ of the $S$. aureus isolates recovered at our institution were methicillin resistant in 2005 and 2006.

The revised guideline for preoperative antibiotic prophylaxis was implemented in April 2004. In January 2003, the rate of compliance with the guidelines for preoperative antibiotic prophylaxis was $29.9 \%$. After instruction was provided and the new guideline was implemented, the compliance rate improved to $54.5 \%$. Aberration in the time of prophylaxis (ie, delayed or premature administration) was the most frequent reason that prophylaxis failed to comply with the guideline. In 2003, preoperative prophylaxis was administered at an incorrect time for $42.3 \%$ of procedures; in 2004, prophylaxis was administered at an incorrect time for $31.0 \%$ of procedures $(P<.001)$. Additional dosages were given to $26.5 \%$ of patients in 2003 and to $2.3 \%$ of patients in $2004(P<.001)$. Prophylaxis was administered without indication for $11.8 \%$ of procedures prior to the intervention, but only for $1 \%$ of procedures after the intervention $(P=$ $.002) .{ }^{13}$

Antibiotic therapy that was not prescribed in accordance with the guideline was prescribed for $93(30.5 \%)$ of 305 patients with bacteremia. For 62 of these patients $(66.7 \%)$, empirical therapy was switched to a narrower-spectrum agent or to a first-line agent. More importantly, $26(27.5 \%)$ of these patients received empirical therapy that was not active against the organism they were known to be infected with, or they received no antibiotic therapy at all. ${ }^{14}$

As an outcome parameter, the prevalence of nosocomial infection at our institution had been documented repeatedly by prevalence studies (Figure). Compared with the mean val-

TABLE 3. Estimates of the Reduction in Costs Associated With Nosocomial Infection

\begin{tabular}{lc} 
Variable & $\begin{array}{c}\text { Estimated value } \\
\text { in } 2006\end{array}$ \\
\hline $\begin{array}{l}\text { No. of nosocomial infections prevented } \\
\text { Cases of bacteremia prevented }\end{array}$ & 700 \\
$\begin{array}{l}\text { Money saved, at a cost of } € 3,000 \\
\text { per nosocomial infection }\end{array}$ & 110 \\
$\begin{array}{l}\text { Estimated cost per year for additional staff } \\
\text { and infrastructure }\end{array}$ & $€ 2,100,000$ \\
Savings per year & $€ 200,000$ \\
\hline
\end{tabular}

NOTE. Values for 2006 are in comparison with the period prior to 2004, that is, the period before the infection control program was implemented. 
ues of studies prior to $2006(11.7 \%)$, the nosocomial infection rate was reduced by $41.2 \%$ after the intervention. Before the intervention, between $10.5 \%$ and $13.0 \%$ of the patients were affected by nosocomial infections. After the intervention, the prevalence decreased to $6.8 \%$. (OR for 2006 compared with 2004, 0.5 [95\% CI, 0.27-0.88]; $P=.01$ ). In 2004, the number of hospital admissions was 19,968 , and the mean length of stay was 8.5 days; in 2006, there were 20,059 admissions and the mean length of stay was 8.5 days. We estimate that this infection control program prevented approximately 700 nosocomial infections at our institution (Table 2). Furthermore, previous 1-week prevalence studies identified between 3 and 11 (mean, 5) cases of nosocomial bacteremia per week until 2004. In 2006, there were only 2 cases per week $\left(\chi^{2}=\right.$ $5.86 ; P=.016)$.

\section{I S CUSSION}

Repeated studies of the prevalence of nosocomial infection in 1999, 2002, and 2003 showed a high prevalence at our institution. Eventually, 1 additional person (a hospital epidemiologist) was employed. Together with the infection control nurse, and in cooperation with the microbiologist and the infectious diseases clinician, an infection control program was implemented. Additional staff members are usually believed to generate additional costs. Therefore, it is difficult to convince stakeholders in hospital administrations to finance infection control programs, because their primary interest is to reduce healthcare costs. ${ }^{15}$ The potential impact of an infection control program is difficult to document, and it is nearly impossible to achieve maximum impact if an infection control team is understaffed. As do most other preventive activities, infection control has to fight against its poor image in the community: prophylaxis itself does not generate profit, and therefore, it is not attractive. It prevents a potentially life-threatening situation for the patient, and it prevents costs for the hospital. The costs that are avoided, however, cannot be factored into an annual budget. The savings only become apparent in comparison with costs from previous years before preventative measures were implemented, when the rate of nosocomial infection was higher. In contrast, it is easy to calculate the costs associated with the employment of infection control staff and the improvement of infrastructure. Communication is crucial to overcoming this conflict between clinicians and administrators, because quality improvement is often cost effective, and cooperation is the way to improve quality of care and reduce costs. ${ }^{6}$ Infection prevention at hospitals is highly cost effective, ${ }^{5,6,16}$ but finances are not the only reason to enhance hygiene measures at hospitals. Ethical concerns, patient safety, and quality-of-care issues are the main reasons. ${ }^{7}$

Isolation of patients-for example, those infected and/or colonized with MRSA-has been criticized as being ineffective and as increasing the risks for patients in isolation because if lowers the quality of care. ${ }^{17,18}$ Cepeda et al. ${ }^{17}$ compared isolation with standard care in a intensive care unit where the rate of hand hygiene compliance was $21 \%$; screening for colonization with MRSA was performed for only approximately $80 \%$ of patients, and patients were not isolated between the time screening samples were obtained and the time culture results were available. Thus, in that study, the spread of MRSA on HCWs' insufficiently disinfected hands could continue for 2 additional days, until culture results became available, or even longer if patients were not screened. The Cepeda et al. ${ }^{17}$ study does not document the ineffectiveness of isolation measures; it shows that if isolation is delayed and adherence to hand hygiene recommendations is low, the transmission of MRSA is not preventable. The combination of improved hand hygiene and preventive isolation during the time between obtaining screening samples and receiving culture results would probably be more effective. ${ }^{19}$ However, this approach is much more feasible at an institution such as our hospital, where there is a low prevalence of infection and colonization with MRSA, than it would be in a setting where MRSA was endemic. The goal of our multimodal intervention was to maintain this low prevalence of MRSA at our institution and, more generally, in our area, and to reduce the transmission of pathogens. During the study period, there was no increase in the incidence of infection or colonization with MRSA. Our intervention may have contributed to this result. Observational studies do not allow for the evaluation of a single intervention, but they may better reflect clinical practice, and hence, be a more appropriate way than a randomized, controlled clinical trial to show whether it is possible to prevent the spread of MRSA in a clinical setting.

Preoperative antimicrobial prophylaxis has been established as an important measure for decreasing the rate of postoperative wound infection. The efficacy of preoperative prophylaxis depends on the time at which it is administered..$^{20}$ It is frequently prescribed for prolonged periods and may contribute to the emergence of resistant pathogens. ${ }^{21}$ The main goal of this step of our intervention was to standardize the prophylaxis procedure and, therefore, increase the compliance with the administration time recommended in the guideline and avoid unnecessary postoperative administration. The distribution of the guideline on pocket cards made it easily available and may have contributed to improved compliance and reduced antibiotic consumption. Webb et al. ${ }^{22}$ showed that improved preoperative use of antibiotics resulted in a significant decrease in surgical site infection. In our institution, we were not able to quantify the effect of our intervention on surgical site infections. However, significantly improved adherence to the prophylaxis guidelines may have had a positive impact.

At our institution, about one-third of BSIs are hospital acquired. ${ }^{14} \mathrm{Up}$ to $19 \%$ of the nosocomial infections documented during prevalence studies were BSIs. In the 1970s, nosocomial BSI had already been shown to cause an excess length of stay of 14 days and $\$ 3,600$ in increased direct costs. ${ }^{23}$ In intensive care units, nosocomial BSI causes attributable 
costs of $\$ 40,000$ per survivor, an excess length of stay of 24 days, and an attributable mortality of $35 \% .{ }^{10}$ The improved management of bacteremia may improve outcome ${ }^{24}$ and reduce costs. Therefore, in our study the infectious disease clinician visited and assessed all patients with blood cultures positive for a pathogen. For approximately one-third of these patients, treatment was modified in accordance with guidelines. This type of intervention is a kind of secondary prevention. Its goal is simply to improve the management of an established nosocomial BSI. However, this intervention may have had a positive impact on outcomes for these patients, as has been shown in a similar setting. ${ }^{24}$

In contrast to other studies, our review of interventions at our institution did not focus on a single intervention. We report on a multimodal approach that represents a real-life situation at a tertiary care center. The most important and effective intervention may have been the hand hygiene campaign; increased adherence to preoperative antibiotic prophylaxis guidelines, supervised isolation of patients colonized and/or infected with multidrug-resistant bacteria, and therapy recommendation for all patients with bacteremia from the infectious diseases clinician probably have only a minor direct impact on the rate of nosocomial infection. However, all of the interventions combined with the frequent presence of infectious diseases and hygiene staff in wards had an impact on the perception of hygiene issues and, therefore, may have indirectly contributed to the reduction of the nosocomial infection rate, which is a reliable outcome parameter. In accord with the observed reduction in the prevalence of nosocomial infection, we observed, as surrogate marker, a 32\% increase in the consumption of alcohol-based hand rub after the intervention. For the first time in the past 10 years, the hospital pharmacy did not record an increase in the consumption of antibiotics, although the number of admissions at our hospital has been continuously increasing. The effect of our infection control program was greater than expected, but there may have been a Hawthorne effect, and the results may have been short lived if the interventions were not continued. To reduce this risk, the program will be continued, and hand hygiene compliance and prevalence rates for nosocomial infection will be studied annually.

We estimate that our infection control program reduced the number of nosocomial infections per year by approximately 700 . Assuming a case-fatality rate of $5 \%$ for nosocomial infection, ${ }^{25,26}$ the program may prevent approximately 25-50 deaths per year. The costs associated with a single nosocomial infection, based on the literature, ${ }^{2-4}$ range between $€ 1,200$ and $€ 6,000,{ }^{2}$ or up to $\$ 40,000$ for BSIs in intensive care units. ${ }^{10}$ The additional length of hospital stay added by a nosocomial infection ranges from 1 to 11 days, and up to 12 days for the $41 \%$ of rehospitalized patients who acquired nosocomial infection during their first hospital stay. ${ }^{26}$ Thus, our infection control program may save approximately $€ 1.9$ million per year (Table 3 ). It seems to be highly cost effective, given that the savings are about 10 times as much as the costs of the program. Our program demonstrates that the reduction of nosocomial infection rates by about one-third ${ }^{5}$ is an achievable goal. The employment of staff qualified to implement such a program should be one of the first goals for clinicians and hospital administrators, not only for economic reasons, but even more importantly, for ethical reasons, and to improve the quality of care and patient outcomes.

Address reprint requests to Thomas Bregenzer, MD, Division of Infectious Diseases, Department of Internal Medicine, Kantonsspital Aarau, 5000 Aarau, Switzerland (thomas.bregenzer@ksa.ch).

Published in part: Sixth International Conference of the Hospital Infection Society; Amsterdam, The Netherlands; October 15-18, 2006 (Abstract P12.40).

\section{REFERENCES}

1. Semmelweis PI. Aetiologie, Begriff und Prophylaxis des Kindbettfiebers. Pest 1861. Reprint. Leipzig: Ambrosius Barth; 1912.

2. Wilcox MH, Dave J. The cost of hospital-acquired infection and the value of infection control. I Hosp Infect 2000; 45: 81-84.

3. Wenzel RP. The economics of nosocomial infections. J Hosp Infect 1995; 31: 79-87.

4. Zhan C, Miller MR. Excess length of stay, charges and mortality attributable to medical injuries during hospitalization. JAMA 2003; 290: 18681874.

5. Haley WR, Culver DH, White JW, et al. The efficacy of infection surveillance and control programs in preventing nosocomial infections in US hospitals. Am J Epidemiol 1985; 121: 182-205.

6. Meliones J. Saving money, saving lives. Harv Bus Rev 2000; 78:57-67.

7. Herwaldt LA. Ethical aspects of infection control. Infect Control Hosp Epidemiol 1996; 17:108-113.

8. O'Boyle C, Jackson M, Henly SJ. Staffing requirements for infection control programs in US health care facilities: Delphi project. Am J Infect Control 2002; 30:321-333.

9. Sax H; Swiss-NOSO. Nationwide surveillance of nosocomial infections in Switzerland-methods and results of the Swiss Nosocomial Infection Prevalence Studies (SNIP) in 1999 and 2002 [in German]. Ther Umsch 2004; 61:197-203.

10. Pittet D, Tarara D, Wenzel P. Nosocomial bloodstream infection in critically ill patients: excess length of stay, extra costs, and attributable mortality. JAMA 1994; 271:1598-1601.

11. Leibovici L, Shraga I, Drucker M, Konigsberger H, Samraz Z, Pitlik SD. The benefit of appropriate empirical antibiotic treatment in patients with bloodstream infections. J Intern Med 1998; 244:379-386.

12. Campagne suisse d'hygiène des mains. Swisshandhygiene.campaign .2005/2006, organized by Swiss-NOSO. Available at: http://cclin-sudest .chu-lyon.fr/antennes/auvergne/Auvergne_journee05/Uckay_05.pdf. Accessed November 21, 2007.

13. Schmid F, Ebnöther C, Heinzer I, Bregenzer T. Can the infectious diseases service improve management of bloodstream infections? In: Programs and abstracts of the 46th Interscience Conference on Antimicrobial Agents and Chemotherapy (Washington, DC). 2005:367. Abstract K1934.

14. Schmid F, Ebnöther C, Wydler J, Bregenzer T. Compliance to guidelines for preoperative antibiotic prophylaxis. In: Program and abstracts of the Swiss Infection Conference (Basel, Switzerland). 2005:36. Abstract p94.

15. Herzlinger RE. Why innovation in health care is so hard. Harv Bus Rev 2006; 84:58-66.

16. Wenzel RP. Does infection control control infections? Schweiz Med Wochenschr 2000; 130: 119-121.

17. Cepeda JA, Whitehouse T, Cooper B, et al. Isolation of patients in single 
rooms or cohorts to reduce spread of MRSA in intensive-care units: prospective two-centre study. Lancet 2005; 365: 295-304.

18. Stelfox HT, Bates DW, Redelmeier DA. Safety of patients isolated for infection control. JAMA 2003; 290:1899-1905.

19. Salaripour M, McKernan P, Devlin R, et al. A multidisciplinary approach to reducing outbreaks and nosocomial MRSA in a university-affiliated hospital. Healthc Q 2006; 9: 54-60.

20. Classen DC, Evans RS, Pestotnik SL, Horn SD, Menlove RL, Burke JP. The timing of prophylactic administration of antibiotics and the risk of surgical-wound infection. $N$ Engl J Med 1992; 326: 281-286.

21. Kernodle DS, Barg NL, Kaiser AB. Low-level colonization of hospitalized patients with methicillin-resistant coagulase-negative Staphylococci and emergence of the organisms during surgical antimicrobial prophylaxis. Antimicrob Agents Chemother 1988; 32:202-208.

22. Webb AL, Flagg RL, Fink AS. Reducing surgical site infections through a multidisciplinary computerized process for preoperative prophylactic antibiotic administration. Am J Surg 2006; 192:663-668.

23. Spengler RF, Greenough WB 3rd. Hospital costs and mortality attributed to nosocomial bacteremias. JAMA 1978; 240:2455-2258.

24. Fluckiger U, Zimmerli W, Sax H, Frei R, Widmer AF. Clinical impact of an infectious disease service on the management of bloodstream infection. Eur J Clin Microbiol Infect Dis 2000; 19:493-500.

25. Wisplinghoff $H$, Bischoff T, Tallent SM, Seifert H, Wenzel RP, Edmond MB. Nosocomial bloodstream infections in US hospitals: analysis of 24,179 cases from a prospective nationwide surveillance study. Clin Infect Dis 2004; 39:309-317.

26. Kirkland KB, Briggs JP, Trivette SL, Wilkinson WE, Sexton DJ. The impact of surgical-site infections in the 1990s: attributable mortality, excess length of hospitalization, and extra costs. Infect Control Hosp Epidemiol 1999; 20: 725-730. 